\title{
Echocardiography in Rheumatoid Arthritis (RA)
}

\author{
Małgorzata Wisłowska \\ Head of Clinic of Rheumatology $\mathcal{E}$ Internal Medicine of Central Clinical Hospital of \\ Ministry of Internal Affairs and Administration in Warsaw, Warszawa
}

Poland

\section{Introduction}

The results of numerous clinical studies confirm the presence of cardiac abnormalities in patients with rheumatoid arthritis. Their authors emphasize the utility of echocardiography in detecting heart muscle damage, pericardial involvement and valvular heart disease in RA. Bacon and Gibson, using one-dimentional imaging mode, found mitral valve changes in $6.9 \%$ of their patients and ascribed it to the systemic spread of the disease. Prakash, Nomeir and MacDonald noted mitral valve defects in 25, 30 and $10 \%$ of RA individuals, respectively. Using two-dimensional technique Mody discovered the same disorder in $13 \%$ of his RA cases and additionally aortic valve insufficiency in a small percentage of this subset. Toumanidis et al. revealed mitral valve and aortic cusps derangements in about $24 \%$ of their RA patients. In Wisłowska's study mitral valve insufficiency was present in $8.6 \%$ of RA patients and occurred more frequently in them than in the controls. One must take into consideration, however, that mitral valve prolaps is observed in up $18 \%$ of healthy individuals, and therefore can not be regarded an RA characteristic.

Echocardiography also revealed discrepancies in heart muscle structure and function between RA patients and the control groups. Wisłowska found left ventricular mass in RA individuals significantly greater then in the controls. The same concerned intraventricular septum end diastolic thickness, LV posterior wall end diastolic thickness and the aortic root diameter. The ejection fraction was significantly lower and isovolumetric relaxation time (IVRT) and deceleration time significantly longer in RA patients compared to the controls. These findings are in accordance with Alpaslan, Di Franco and Levendoglu's results, that revealed significant differences in LV diastolic function (peak E velocity, E velocity/A velocity ratio, IVRT, myocardial performance index [MPI] and transmural flow propagation velocity [TFPV]) between RA group and the control subjects. The results of these studies indicate to the presence of subclinical myocardial involvement in RA, which can be ascribed to nonspecific myocarditis observed in this disease. Nevertheless different other risk factors for cardiac muscle impairment are usually present in RA individuals and therefore it is uncertain, whether heart pathology in rheumatoid arthritis is due to inflammation itself or is secondary to other process or to drug use in this disease.

Although pericardial effusion is considered the most common heart complication in RA, Wisłowska et al. observed it only on $4 \%$ of cases in echocardiography image. Pathologists find it in about $30 \%$ of RA cases post mortem, but clinical manifestation of pericarditis is rare in this disease. It's life-threatening complications such as constrictive pericarditis or tamponade were reported in very few RA cases, to date. 
Rheumatoid arthritis (RA) is a connective tissue disease predominantly affecting joints and periarticular structures. Inflammatory process within the skeletal system - a source of patients' main complaints and eventual disability - draws the attention of medical service to the extent that sometimes results in negligence of other aspects of the systemic disorder. These in turn can be of importance, since inflammation in RA, as in other connective tissue diseases is widespread and affects such vital tissues and organs as those of the cardiovascular system, for instance. Extraarticular manifestations in RA however develop slowly and are poorly manifested. Their symptoms tend to be are assigned rather to patient's general malaise and the lack of fitness. Heart involvement is often asymptomatic or causes mild ailments, frequently disregarded by affected persons, because RA individuals are generally not energetic and avoid to move too much. Chest pain or fatigue in them happens to be contributed to skeletal system involvement rather then to other pathological process. These are perhaps the reasons why at least twice as many changes in RA hearts are recognized post-mortem then during the patient's' lifetime. One of the current issues in rheumatology therefore is to recognize the presence, type and the extend of heart involvement in RA and to search for correlations of their appearance and intensity with the disease clinical picture. Echocardiography appears to be of much help in this aspect. The development of this domain improved cardiological diagnostics, providing a valuable tool to assess the condition of heart structures and their functional properties. It enabled detecting even minor cardiac muscle, valvular and pericardial aberrations, also in asymptomatic individuals. Based on these observations numerous clinical studies to date focused on echocardiography application in RA and it's utility in diagnosing cardiovascular complications of the disease.

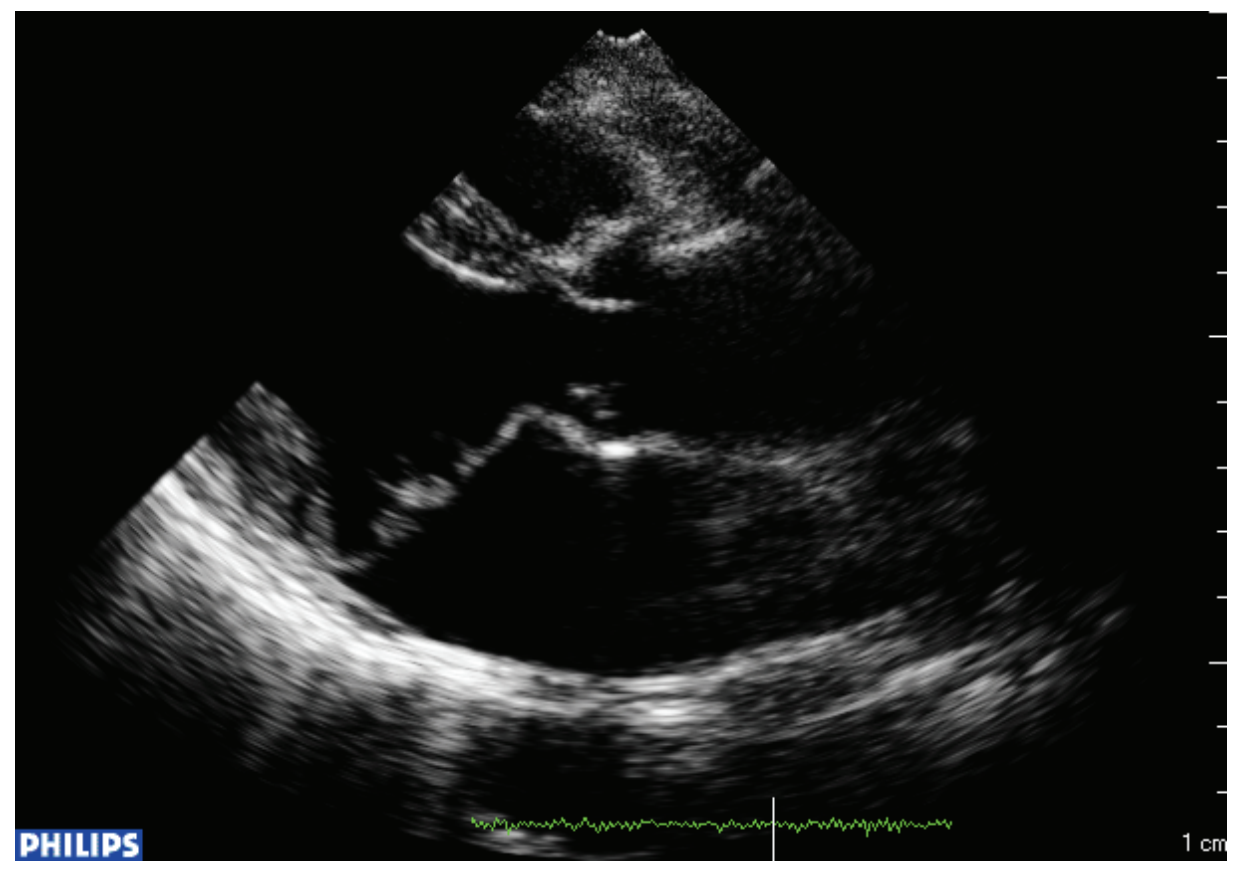

Fig. 1. Echocardiogram of RA patient LP female, age 46 - mitral valve prolaps 
One of the firsts to examine echocardiography images RA patients were Bacon and Gibson [1]. In their one-dimentional examination of 44 individuals with classical or definite RA - 22 with palpable rheumatoid nodules and 22 without them - they demonstrated the presence of pericardial effusion in $34 \%$ of patients. It was detected in $50 \%$ of those with nodular and in $18 \%$ of those with non-nodular disease course. In most cases the fluid did not cause symptoms of pericarditis, although in several patients it was $1-2 \mathrm{~cm}$ in depth. No correlation could be seen between the appearance of effusion and the disease duration and pericardial pathology was not detected in age-matched controls with noninflammatory osteoarticular pathology. Bacon and Gibson also found mitral valve changes in $6.9 \%$ of their RA patients. In their study the average mitral diastolic closure rate in the control group was $116 \mathrm{~mm} / \mathrm{s}$ (range 75 to 180; normal range over $80 \mathrm{~mm} / \mathrm{s}$ ). It was significantly lower in nodular RA group $(63 \mathrm{~mm} / \mathrm{s})$, then in the non-nodular one $(\mathrm{p}<0.05)$ and in the controls $(\mathrm{p}<0.001)$, and only in 5 nodular patients the values were normal. In 3 nodular patients they were markedly decreased (less then $30 \mathrm{~mm} / \mathrm{s}$ ), symptomatic for severe rheumatic stenosis. In non-nodular group mean mitral diastolic closure rate was $94 \mathrm{~mm} / \mathrm{s}$ (range 40-200) and only in 4 cases the values were below normal range. The frequency of diminished rate of mitral valve movement in this study increased with age in RA individuals, but not in the controls and increased with RA duration. The recording of a diminished rate of spontaneous mitral valve movement during the mid-diastolic partial closure period was a new observation. The correlation of decreased mitral diastolic closure rate with the duration of the disease, it's coincidence with severe nodular process and pericardial effusion was, according to authors, a strong argument for considering this parameter representative for a systemic RA manifestation.

Other investigators in 70-ties (Prakash et al. [2], Nomeir et al. [3] and MacDonald et al. [4]) reported mitral valve derangements in 25,30 and $10 \%$ of RA patients, respectively. Prakash et al. [2] performed a prospective echocardiography study to search for cardiac abnormalities in 1 female and 15 male American patients (11 white and 5 black persons), free of cardiac symptoms. $44 \%$ of them had evidence of posterior pericardial effusion, not detected by electrocardiogram nor by the chest X-ray study. The E to F slope of the anterior leaflet of mitral valve was reduced in $25 \%$ of patients, which according to authors, could be due either to rheumatoid involvement of the mitral valve cusps or to changes in left ventricular compliance, both common in people with long-standing rheumatoid arthritis. The study revealed high prevalence of mitral valve and pericardial involvement in RA patients, previously only found in post-mortem studies and confirmed the utility of echocardiography in RA diagnosis.

In 1973 Nomeir et al. [3] studied the nature and extent of cardiac involvement in RA patients. Physical examination of 30 Americans with classic RA did not show any significant cardiac abnormalities, while echocardiography revealed pericardial effusion in $46.6 \%$ and mitral valve defects in $30 \%$ of patients, therefore demonstrating the presence of one of these pathologies in quite a large number of RA individuals. Although there was no correlation between different modalities of the study, echocardiography was claimed by the authors the most sensitive method of detecting cardiac complications of RA at early disease stage.

In 1975 in turn, Davia et al. [5] examined 35 consecutive American RA patients, submitting them to echocardiography to evaluate the motion of the anterior mitral valve leaflet. In contrast to previous results, through analysis they revealed normal valve motion and a normal E/F slope in as many as 31 of them. The authors discovered, that falsely low E/F slope values were noted only when the technique applied was not sufficiency meticulous, 
while the improvement of method resulted in normalization of these values. Their conclusion therefore was that anterior mitral valve leaflet abnormalities rarely if ever are seen in RA patients, provided that careful attention to recording technique is observed.

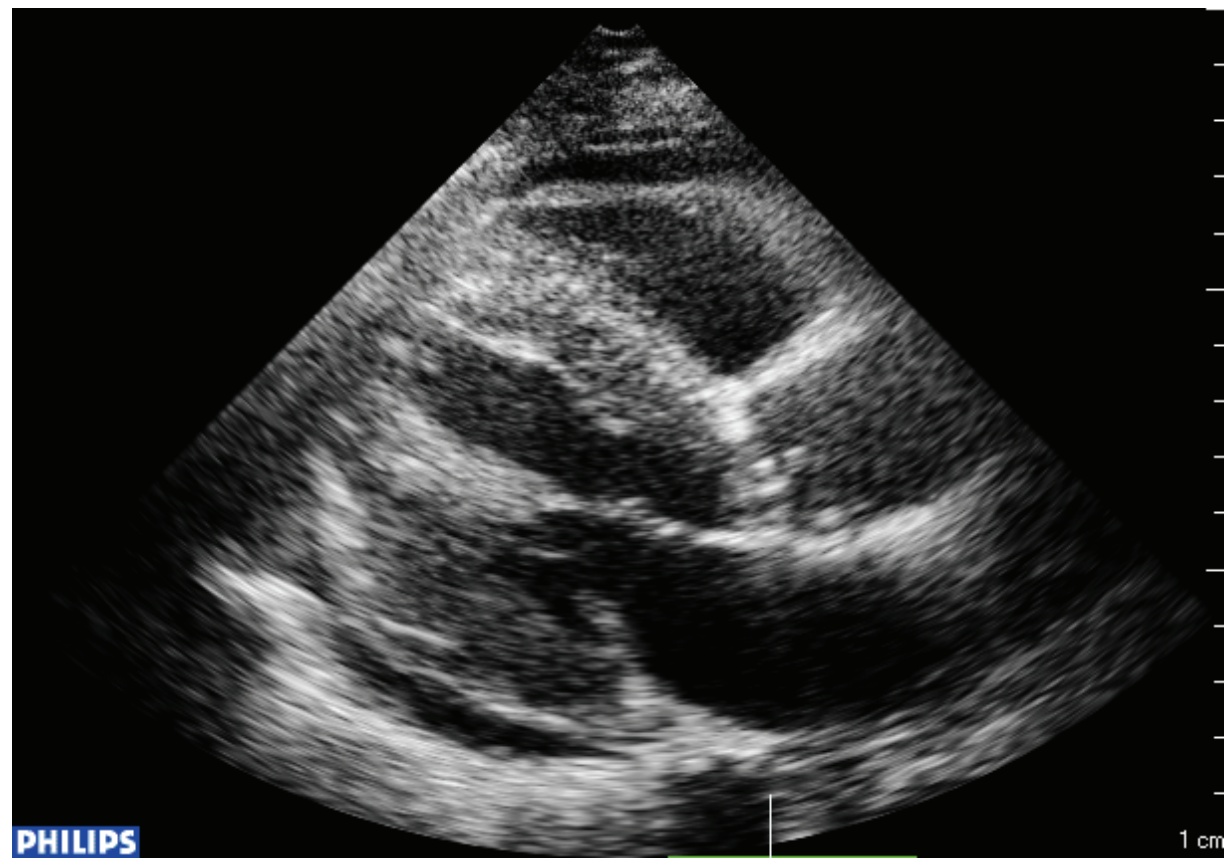

Fig. 2. Echocardiogram of RA patient AM female, age 58 - pericardial effusion

The results obtained by Nomeir et al. [6] in their subsequent study initially showed that 18 out of 30 their RA patients had cardiac involvement arising from the disease at baseline of the examination [3]. Abnormalities detected by echocardiography comprised mitral valve disease or pericardial alterations, or both. All patients were then followed up for 4 years from the initial workup. At the end of the study mitral valve abnormalities were still seen in $63 \%$ of those in whom they were previously present and pericardial effusion remained in $20 \%$. Pericardial thickening persisted in 6 out of 7 patients. There was no definitive correlation between the protraction of these abnormalities and other clinical data, but it was noticed that patients who retained pericardial effusion and mitral valve abnormalities showed a higher number of joints involved and a higher erythrocyte sedimentation rate. It is worth mention that none of the patients developed constrictive pericarditis nor the heart failure and that all abnormalities detected remained clinically insignificant. The results of the study suggest that an impressive number of RA patients suffer from cardiac abnormalities related to their disease which may be clinically undetectable and are rarely life- threatening or requiring surgery support.

MacDonald et al. [4] performed clinical, electrocardiographic (ECG) and echocardiography examinations in 51 American outpatients with RA. 31\% of patients had echocardiographic evidence of pericardial effusion and in 2 patients pericardial thickening was demonstrated. Mitral valve E/F slope was normal in as many as 46 out of 51 persons and left ventricular 
performance was depressed only in a few cases. The authors concluded that in unselected outpatients with RA pericardial abnormalities detected by echocardiograph are common although usually clinically unapparent.

Toumanidis et al. [8] visualized mitral and aortic cusps changes in about $24 \%$ of their patients.

In the Hungarian study of Nagyhegyi et al. [9] 100 of patients suffering from ankylosing spondylitis (AS) and 100 patients with RA were examined by clinical, non-invasive cardiological, radiological and laboratory methods to determine the prevalence of cardiac and cardiopulmonary disorders. 14 patients with AS and 24 with RA had several valvular abnormalities. Among those without valvular defects, myocardial systolic dysfunction was detectable in 15 AS and 11 RA cases and cor pulmonale was diagnosed in 16 and 7 patients, respectively. Conduction disturbances were demonstrated in 17 AS and 14 RA individuals.

Mody et al. [7] from South Africa examined their Negro-Caucasoids patients with RA seen in the rheumatic diseases unit during a 16-months period preceding the study. The authors aimed at determining the prevalence of cardiac abnormalities in RA. They used random tablets to select a group out of 330 persons and finally 101 of RA individuals underwent clinical and echocardiography examination. Adequate two-dimentional assessments were obtained in 84 patients and adequate M-mode recordings in 77.31 patients $(37 \%)$ had 45 echocardiographic abnormalities, in 5 patients $(6 \%)$ pericardial effusion was detected. 11 abnormalities of mitral valve noted in $10(13 \%)$ patients: 3 had mitral valve prolaps, 1 - aortic incompetence and flutter on the mitral valve, in 5 patients mitral annular calcium was detected and 1 patient had hypertrophic obstructive cardiomyopathy and mitral calcium deposits. The reduction in E/F slope was noted in 12 patients, 7 of whom had associated cardiac disease, 1 patient had sinus tachycardia and 4 (5\%) - a mild reduction of E/F slope without any other cardiac abnormality. The authors concluded that apart from the presence of pericardial effusion in $6 \%$ and minor abnormalities of the E/F slope in 5\% of patients, all other significant echocardiographic abnormalities could be related to the presence of associated cardiac disease.

In 1983 Villecco et al. [10] from Italy confirmed the utility of echocardiography in detection of cardiac lesions in RA patients. In order to verify the frequency and the extend of heart pathology, the authors performed mechanophonocardiographic studies and simultaneous mono- and bi-dimentional echocardiography in 28 RA individuals. They showed an increase in the PEP/LVET ratio on the polycardiogram in 1 case and echocardiographic alterations in $18(64.3 \%)$ of patients. Pericardial effusion was noted in $21.4 \%$, the thickening of pericardium in $14.3 \%$, alterations of mitral valve (the reduction in protodiastolic closing velocity of the anterior edge of the large mitral valve) in $35.7 \%$ and thickening of the interventricular septum in $17.9 \%$ of cases. The authors reckoned all measurements results to be good indicators of cardiac complications in RA. According to them such examinations allow to identify a group of patients suffering from abnormalities otherwise undetectable.

Wisłowska et al. [11,12] in Poland observed higher frequency of valvular heart disease, especially mitral insufficiency in RA patients than in the control group. Echocardiographic examinations were carried out in 100 patients (age<65 years) with rheumatoid arthritis (stages II-IV according to Steinbrocker's criteria). The control group consisted of 100 patients with osteoarthrosis, spondyloarthrosis and painful shoulder matched for age, sex and body surface area (BSA). All patients with myocardial infarction, hypertension, rheumatic fever or a history of diabetes were excluded from the study. Echocardiographic examination revealed more cases of valvular heart disease, especially mitral insufficiency, in 
RA patients then in the control group. Mitral valve prolaps was noted in $6 \%$ of RA individuals and pericardial effusion in $4 \%$, whereas it was absent in the control group and in all patients during of clinical assessment prior to echocardiography imaging. In all $4 \%$ the volume of fluid was less then $300 \mathrm{ml}$, which was considered neglectable. Patients with RA had greater diastolic left ventricular diameter and aortic root diameter, as well as a smaller ejection fraction, mean velocity of cimcumferential fibre shortening and fractional shortening than the control individuals. The comparison of results of the echocardiographic measurements on different RA stages or in relation to the functional index, seropositivity and seronegativity and in person of different disease duration did not reveal any statistically significant discrepancies. In $39 \%$ of RA patients and 19\% of the controls valvular heart disease was discovered by echocardiographic examination $(\mathrm{p}<0.05)$. Mitral valve insufficiency (I and II degree) was noted in $29 \%$ of RA patients and in $10 \%$ of the controls $(\mathrm{p}<0.005)$, aortic insufficiency (I and II degree) in 8 RA patients and 4 of the control group (NS), tricuspid insufficiency in 3 RA individuals and in 2 control subjects (NS) and mitral stenosis in 1 RA patient. In total, valvular heart disease was seen in 47 RA cases and in 19 control subjects. Only in $4 \%$ of cases pericardial effusion was detected, though this abnormality is considered the most common heart complication in RA.

There is much controversy in literature, regarding the frequency of mitral valve prolaps in general population, where it fluctuated from $3-8 \%$ up to $18 \%$ in different clinical studies $[13,14]$, as well as in RA patients [6]. In Wisłowska's nodular group the prevalence of this abnormality was $8.6 \%$ [12]. Such discrepancies make impossible to classify it as an RA characteristics.

In another study Wisłowska et al. [12] compared the results echocardiographic examinations in 35 patients with nodular and 35 with non-nodular RA. The groups were matched for age, sex and the BSA. 35 patients with osteoarthrosis and spondyloarthrosis matched with both RA groups formed a control group. Patients with history of myocardial infarction, hypertension, rheumatic fever and diabetes were excluded from the study. Echo-Doppler cardiography and echocardiographic Holter monitoring revealed cardiac involvement in $71.9 \%$ of patients with nodular RA and $42.9 \%$ of non-nodular cases compared to $22.9 \%$ of the control group $(\mathrm{p}<0.0002)$. Echocardiographic examination revealed more cases of valvular heart abnormalities, especially mitral insufficiency, in nodular RA patients than in non-nodular and the controls. Both mitral valve prolaps and pericardial effusion were noted in $8.6 \%$ of nodular subjects. Patients with nodular RA also had greater aortic root diameter, a smaller ejection fraction, and smaller mean velocity of circumferential fiber shortening and fractional shortening compared to ones non-nodular and to the control group subjects.

In another study concerning systolic and diastolic heart function in RA patients Wisłowska et al. [15] found left ventricular (LV) mass index in RA significantly greater than in the control group. The same concerned intraventricular septum end-diastolic thickness, LV posterior wall end-diastolic thickness and the aortic root diameter. The ejection fraction in RA patients was significantly lower than in the control group. The assessment of diastolic function parameters revealed significantly longer isovolumetric relaxation time and deceleration time in RA patients.

Similar results were presented by Alpaslan et al. [16], Di Franco et al. [17], and Levendoglu et al. [18]. In their studies echocardiographic indices of LV diastolic function (peak E velocity, E velocity/A velocity ratio, isovolumetric relaxation time [IVRT], myocardial performance index [MPI] and transmural flow propagation velocity [TFPV]) in RA group 
were significantly different from those of the controls. The reported finding indicate to the presence of subclinical myocardial involvement in RA, which is most probably due to the nonspecific myocarditis observed in these patients. Such myocardial involvement was elsewhere described as asymptomatic, rarely influencing cardiac size or function, predominantly affecting LV diastole, usually of diffuse pattern and scarcely clinically significant. It is not know to date, however, to what extent cardiac pathology in RA emerge from inflammatory myocarditis itself or is secondary to other pathology or drug use in this disease.

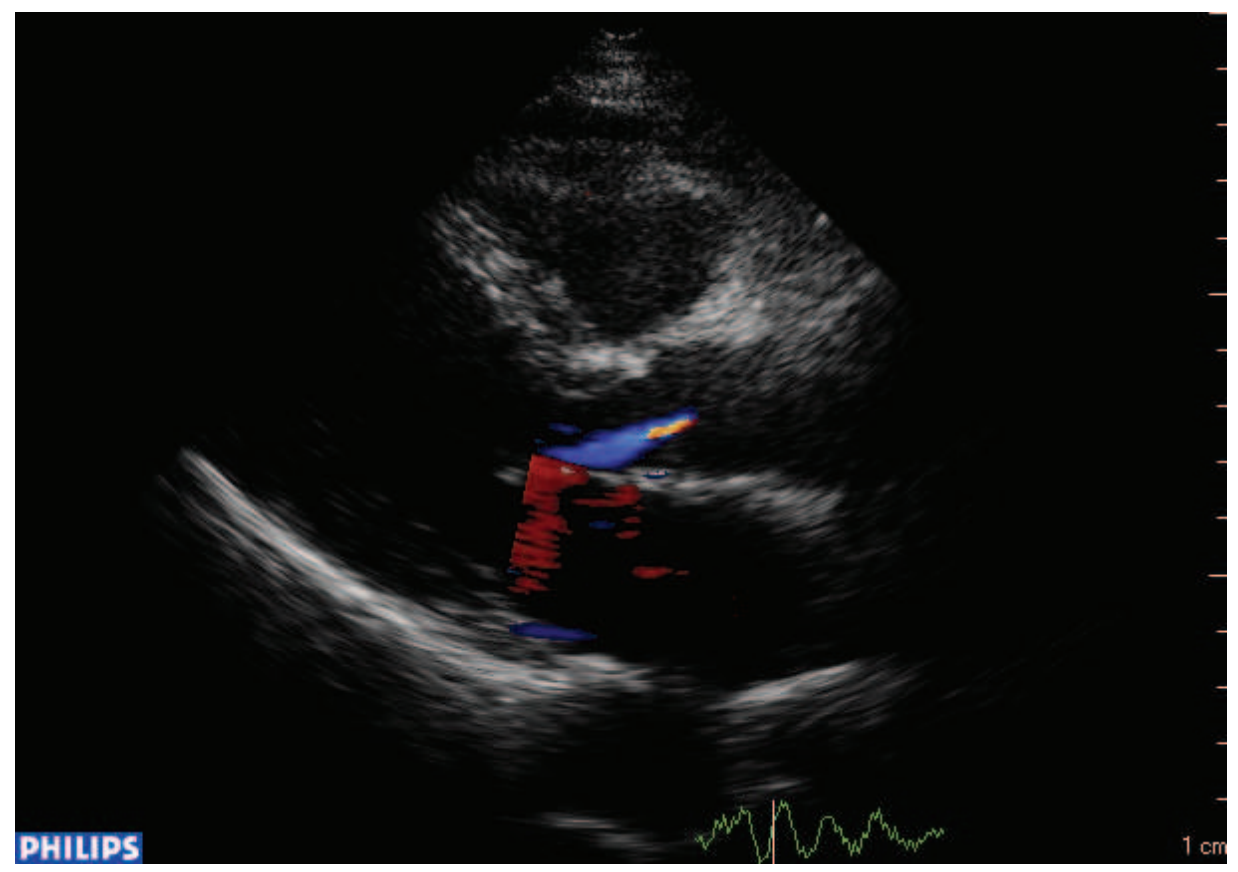

Fig. 3. Echocardiogram of RA patient BS female, age 50 - aortic valve insufficiency

Toumanidis et al. [8] examined a Greek population to evaluate early morphological and functional heart abnormalities. The examined group was free of risk factors for coronary artery disease and without any clinically evident cardiac manifestations. Echocardiography examination was performed in 62 patients with collagen disease, 15 with rheumatoid arthritis and in 40 healthy individuals. The imager was taken from apical four-chamber view at rest and during the end of a 3- minute isometric exercise with handgrip. Global and regional ejection fraction $(\mathrm{EF})$ of left ventricle were calculated. In the RA group the EF was $62.38 \pm 6.88 \%$ vs $61.47 \pm 8.52 \%$ in the controls. Regional EF analysis revealed a major dysfunction of the proximal segment of interventricular septum in all groups and reduced separation of mitral and aortic valve leaflets in RA.

In Maione et al. [19] echocardiography examination of consecutive 39 Italian RA and 40 control subjects the occurrence of anatomic lesions was lower then that observed in other studies. No differences in mean values of left and right ventricular diastolic function indices obtained by Doppler echocardiography were found between patients and the controls. 
However, in $26 \%$ of patients with RA, left ventricular abnormalities, probably secondary to myocardial fibrosis were detected. Pericardial effusions (less than $300 \mathrm{ml}$ ) were found in 3 RA patients (7\%) and in none of the controls. Pericardial adhesion was seen in 1 RA patient only. Valvular involvement (1 case of mitral stenosis and 1 case of aortic stenosis) was detected in 2 RA cases, both having had suffered from rheumatic heart disease in the past. Moreover, the prevalence both: minor changes of mitral valve and trivial regurgitation was similar in the investigated groups. Left atrial or left ventricular dilation was found in $5 \mathrm{RA}$ patients out of whom 3 were affected by coronary artery disease and 2 by valvular disease and in none of the control subjects. Left ventricular hypertrophy was present in 2 RA patients, both suffering from essential hypertension and in none of the controls. EF was normal in all except 3 RA patients, all of whom with ECG evidence of previous myocardial infarction. To investigate diastolic heart function the observers excluded persons with arterial hypertension, diabetes and valvular heart disease (4 RA cases and 1 from the control group). To further exclude the presence of occult heart disease patients and the controls underwent an echo-dobutamine provocative test. Afterwards echocardiographic indices of LV and RV diastolic function were calculated in 35 RA and 39 control subjects. The authors found no statistically significant differences in mean values of the parameters examined. Nevertheless diastolic function abnormalities consisting of IVRT prolongation and inverted E/A ratio were pointed out in 9 out of 35 (26\%) RA cases and in 2 out of $39(5 \%)$ controls. The difference was statistically significant (Fisher exact test, $\mathrm{p}=0.019$ ) and considered due to the decrease in peak early diastolic velocity of the mitral valve. An inverted E/A ratio of the tricuspid flow velocity profile was detected in 3 RA patients, all with LV filling abnormalities, and was not detected in the controls.

Di Franco et al. [17] studied an Italian population to evaluate the LV filling parameters by the analysis of transmitral flow and pulmonary venous flow with special regard to patients' age and the disease duration. 32 RA cases without evidence of concomitant cardiac disease were selected and compared to matched control subjects. All patients and the control group were submitted to M-mode, two-dimensional Doppler and color Doppler (continuous and pulsed wave) echocardiography. Diastolic parameters evaluated comprised transmitral flow (E/A ratio), pulmonary venous flow (S/D ratio), a-Pw, IVRT and deceleration time (DT). In RA LV filling disturbances were apparent and characterized by a reduced E/A ratio (mean SD 1.16 [0.31] vs 1.37 [0.32] in the controls) $(\mathrm{p}=0.02)$ and increased S/D ratio (1.43 [0.40] vs $1.22[0.29]$ in the controls) $(p=0.017)$. In this group of patients the correlation between $\mathrm{E} / \mathrm{A}$ ratio and the disease duration ( $\mathrm{r}=0.40, \mathrm{p}=0.01$ Spearman rank correlation) was also noted. The authors concluded that RA patients, in absence of overt heart disease, show diastolic function characterized by impaired E/A and S/D ratio. According to them it's relation to the disease duration suggests the presence of subclinical myocardial involvement.

In another Italian study Corrao et al. [20] investigated and verified diastolic abnormalities in rheumatoid patients, without clinically evident cardiovascular disease and other confounding complaints, by using pulsed Doppler examination of transmitral blood flow. They selected 40 patients fulfilling revised American Rheumatism Association (ARA) criteria for the diagnosis of rheumatoid arthritis with no symptoms of cardiac disease, nor clinical findings of other extracardiac pathology, matched with 40 healthy volunteers as a control. An echocardiographic examination was carried out in each subject. LV structural and functional measurements were obtained. Intraventricular septum thickness and LV 
mass index were significantly higher in rheumatoid patients than in the control. RA patients had higher mean values of peak $\mathrm{A}$ velocity and $\mathrm{A} / \mathrm{E}$ ratio. When multiple linear regression data analysis was performed, an independent relationship only between A/E ratio and LV mass was found. The results confirmed the presence of diastolic abnormalities in rheumatoid patients and pointed out that these abnormalities also affect echo-Doppler parameters of LV filling. The study results further suggest that structural LV changes can be responsible for LV filling impairment.

One more Italian study was carried out by Montecucco et al. [21] who used digitized Mmode and Doppler echocardiography to assess LV function in 54 patients (mean age 50 years) suffering from active RA, without obvious cardiovascular disease. The group was compared with 54 age and sex matched normal subjects. No differences were found in LV end-diastolic diameter, systolic function and parietal thickness between patients and the controls. However, a significant reduction of various indices of LV diastolic function was recorded, including E/A ratio and the peak lengthening rate of the LV diameter (an index of relaxation evaluated by M-mode echocardiography). The former was correlated with patients' age and was independent of disease duration while the latter was more markedly correlated with the disease duration then with the patients' age. The authors suggested that the observation of relationship between diastolic impairment and the disease duration in active RA may open new perspectives for the study of RA-associated cardiovascular disease. Voipio-Pulkki and Saraste [22] examined a Finish RA population. LV function was studied in 182 outpatients with rheumatoid arthritis compared with 182 controls matched for age and sex. Patients with RA showed mild but definite tachycardia and lower systolic and diastolic blood pressures at rest. PEP/LVET was equal in both groups and LV mass assessed by echocardiography tended to be increased in males with seropositive disease, while no differences were found in ejection fractions. Mean velocity of cimcumferential fibre shortening (VCF) was significantly higher in female patients then in the controls. As VCF corrected for heart rate showed no difference between patients and controls, this apparently reflected an adequate response to the higher pulse rate. Taken together, the results do not support the concept of LV dysfunction in chronic RA, but rather an altered haemodynamic state caused by the disease itself or by it's treatment.

The next Finish study performed by Mustonen et al. [23] concerned cardiac performance in 12 asymptomatic male patients with RA and 14 control subjects. It was planed to elucidate early disturbances in cardiac function in these subsets. In echocardiography, an IVRT and peak filling rate lower in RA patients then in the controls, which apparently reflected an impairment in LV diastolic function. LV diastolic function assessed by radionuclide angiocardiography at rest and during exercise was similar in both groups. There were no differences between the patients and the control subjects, as regards the heart rate, systolic blood pressure and oxygen uptake during peak exercise. LV diastolic function was impaired in spite of normal LV systolic function in RA patients without clinically evident cardiovascular disease and the authors suggested that the excess of cardiovascular mortality in RA patients can be ascribed to this phenomenon. They emphasized the importance of further epidemiological studies to elucidate this matter.

Kozakova et al. [24] performed echocardiographic, electrocardiographic and roentgenologic examinations in 50 Czech patients with RA and no clinical signs of pericardial effusion. Using one-dimentional echocardiography, pericardial effusion was detected in $27(54 \%)$ subjects. Neither valvular involvement nor specific changes in myocardium were found. In sera of patients with pericardial effusion the presence of rheumatoid factor was significantly 
more frequent then in patients without effusion. Patients on steroid therapy for the primary disease had statistically lower incidence of pericardial involvement than patients who were subjected to other forms of treatment. Again, echocardiography proved to be the only sensitive non-invasive method capable of detecting small and medium-sized effusions in pericardial cavity.

In the next Czech echocardiography study of Alusik and Skalicka [25] pericardial effusion was detected in 35 out of 104 RA patients. The amount of fluid was small in 29 , medium in 3 and large in 3 of them. The thickening of pericardium was seen in 4 patients, mitral valve prolaps in 22 and thickened mitral valve in 10. In 24 patients the authors found a small regurgitation at the mitral valve in 10 patients it was more significant. Thickened aortic valve was seen in 14, a small regurgitation though the valve in 8 and a significant one in 4 patients. The LV dilatation was detected in 13 subjects, it's hypertrophy in 12 and an impaired kinetics in another 12 individuals. Dilatation of the RV was seen in 15 and it's hypertrophy in 3 patients. Ventricular hypertrophy and dilatation as well as an impaired LV were interpreted as consequences of valvular involvement and of associated diseases.

Alpaslan et al. [16] examined a Turkish population of RA patients to assess ventricular function by measurement of myocardial performance index (MPI) and transmitral flow propagation velocity (TFPV), which they reckoned better indices of ventricular function than hithero utilized pulsed-Doppler echocardiography of LV inflow, the results of which were affected by changes in pre- and afterload, tachycardia or first degree A-V block as well as pseudonormalisation phenomenon. 32 patients with long-standing RA and 32 control subjects participated in the study. Systolic function was assessed by subjective evaluation of wall motion for both ventricles and by the assessment of fractional shortening of the LV. LV diastolic function was evaluated by standard pulsed-wave Doppler echocardiography, the MPI and the TFPV. RV function was evaluated by MPI. No subject has signs nor symptoms of clinically overt heart failure. Systolic function was normal in all of them. Echocardiographic functional indices of LV diastolic performance: the peak E velocity, E/A velocity ratio, IVRT, MPI and TFPV in the RA group were significantly different from those of the controls $(\mathrm{p}<0.05)$. However, the authors did not observe any significant difference in RV echocardiographic indices between the two groups. Their results show, the presence of LV diastolic dysfunction in patients with long-standing RA. The lack of history of cardiotoxic antirheumatic drug use suggested that this abnormality was due to RA itself. The study showed that LV MPI and TFPV, both easily obtained and not affected by ventricular geometry, well identify the presence of LV diastolic dysfunction in patients with long-standing disease. The findings were in accordance with the results of classic measurements methods of LV function: decreased E/A ratio and prolonged IVRT.

Levendoglu et al. [18] in the study on Turkey population tried to evaluate cardiac involvement in patients with active rheumatoid arthritis. 40 of them participated in the study. All were submitted to standard Doppler echocardiography and MPI grading, which revealed LV and RV diastolic function abnormalities with LV MPI significantly higher than in controls $(\mathrm{p}<0.05)$. A relationship was also found between LV E/A ratio, IVRT and the disease duration $(r=-0.47$ and $p=0.002, r=0.618$ and $p=0.000$, respectively). Diastolic function was impaired in both ventricles and a direct relationship between some of the parameters of LV diastolic function and the disease duration was observed, too. The findings suggest the presence of subclinical myocardial involvement in RA patients. The earliest deterioration in cardiac disease is an impaired diastolic function. Such abnormalities have been reported in a number of conditions such as arterial hypertension, coronary artery disease and in elderly 
subjects, yet the authors selected patients without no evidence of heart problems and impaired ventricular function was observed at all ages in RA, contrary to the control group. Another Turkish study performed by Arslan et al. [26] was planned to evaluate LV diastolic function in patients with active RA by the analysis of conventional Doppler and tissue Doppler echocardiographic imaging (TDI). 52 patients with active RA and 47 healthy persons were included in this study. All were evaluated by M-mode, two-dimentional conventional Doppler echocardiography and the TDI. Late diastolic flow velocity (A) and deceleration time (DT) values were higher in patients with RA than that in the control group $(p<0.001)$ and E/A ratio was lower in RA than in the controls $(p<0.001)$. Among TDI parameters mitral annular early diastolic velocity $(\mathrm{Em})$ and Em/Am (mitral annular late diastolic velocity) ratio were lower in RA patients then in the control group $(p<0.001)$. The correlation was found between A $(r=0.43, p=0.001)$, DT $(r=0.30, p=0.03)$, E/A ratio $(r=0.409$, $\mathrm{p}=0.004), \mathrm{Em}(\mathrm{r}=0.32, \mathrm{p}=0.02), \mathrm{Em} / \mathrm{Am}$ ratio $(\mathrm{r}=0.30, \mathrm{p}=0.03)$ and $\mathrm{E} / \mathrm{Em}(\mathrm{r}=0.32, \mathrm{p}=0.02)$ and the RA duration. The authors concluded, that patients with active RA in absence of clinically evident heart disease show diastolic dysfunction characterized by impaired E/A ratio, $\mathrm{Em} / \mathrm{Am}$ ratio and the DT. The relation between diastolic dysfunction and the disease duration suggests a subclinical myocardial involvement.

The Turkish study of Rexhepaj et al. [27] was aimed the assessment of prevalence of diastolic LV and RV dysfunction in patients with rheumatoid arthritis. The authors tried to estimate whether there is a correlation between the duration of RA and the degree of LV diastolic failure. 81 patients (61 females and 20 males) with RA and without clinically evident heart disease (group 1) and 40 healthy subjects (29 females and 11 males) (group 2) were matched for age and sex. Echocardiographic and Doppler studies revealed significant differences in E, A and E/A ratio $(0.68 \pm 0.19 \mathrm{~m} / \mathrm{s}$ vs. $0.84 \pm 0.14 \mathrm{~m} / \mathrm{s}, \mathrm{p}<0.001 ; 0.73 \pm 0.15 \mathrm{~m} / \mathrm{s}$ vs. $0.66 \pm 0.13 \mathrm{~m} / \mathrm{s}, \mathrm{p}=0.01$; and $0.97 \pm 0.3$ vs. $1.32 \pm 0.37, \mathrm{p}<0.001$, respectively) between RA patients and the controls. Significant difference between groups was also noted in RV early diastolic (Er)/atrial (Ar) flow velocities (Er/Ar ratio) $(1.07 \pm 0.3$ vs. $1.26 \pm 0.3, p=0.002)$ and weak correlation was observed between transmitral E/A ratio and the duration of RA $(\mathrm{r}=-0.22, \mathrm{p}=0.001)$. The MPI appeared to differ little in patients with RA as compared to the control group $(0.51 \pm 0.1$ vs. $0.52 \pm 0.2, p=N S)$. In RA individuals without clinically evident cardiovascular disease the LV and RV diastolic function were reduced. LV wall thickness, it's dimensions, systolic function and the MPI remained normal.

Another Turkish investigators Yazici et al. [28] suggested that RA is associated with increased risk of cardiovascular mortality. Considering that cardiovascular findings are mostly subtle and diastolic function abnormalities are one of the earliest manifestations, they aimed at determining diastolic abnormalities in RA at baseline and after a 5-year follow-up. 72 RA patients without any known cardiac disease and 67 controls were recruited for the study. Disease activity score (DAS), blood lipids and C-reactive protein (CRP) levels were determined. LV mass, IVRT, E and A were determined by M-mode two-dimensional color Doppler echocardiography. Mitral annular early $\left(\mathrm{E}^{\prime}\right)$ and late $\left(\mathrm{A}^{\prime}\right)$ diastolic velocities were also checked by TDI. 24 RA patients were reevaluated after 5 years for DAS, biochemical variables and echocardiography. In 57 out of $72(76 \%)$ of them and in 12 of the $67(18 \%)$ controls diastolic dysfunction (DD) was diagnosed. In 7 out of 10 patients with DD at baseline it remained in the follow-up, while in 8 patients normal diastolic function sustained. DAS and lipid values did not altered during the follow-up and CRP levels decreased significantly $(\mathrm{p}<0.05)$. In conclusion, RA patients are prone to develop diastolic function abnormalities in comparison to healthy controls. A 5-year follow-up of this group 
showed that although clinical response was unsatisfactory, cardiac function was conserved without a major deterioration. Moreover, disease-modifying antirheumatic drugs (DMARDs), such as anti-TNF alpha agent, did not seem to have a major adverse effect on cardiac findings in patients.

The Turkish study of Birdane et al. [29] was aimed at the comparison of standard Doppler and TDI results in RA patients. In 60 such individuals with longstanding disease LV and RV parameters were assessed by standard pulsed-wave Doppler echocardiography, the color M-mode flow propagation velocity and the TDI. LV TDI was achieved at 4 different sites (lateral, septal, anterior and inferior walls) and RV TDI - through the tricuspid lateral annulus. The analysis of the results showed that the basal clinical and echocardiographic parameters: E, A, diastolic velocities of atrioventricular valves, E/A ratio and pulmonary venous Doppler parameters were similar in both groups. The values of LV and RV $\mathrm{E} /$ wave deceleration times and IVRT of RA patients were greater then in healthy controls $(\mathrm{p}<0.05)$. RA patients also had significantly lower color $\mathrm{M}$-mode flow propagation velocity $(p<0.05)$, while $S^{\prime}$ peak and $E^{\prime}$ peak, two of the LV and RV parameters were similar in both groups. Furthermore $A^{\prime}$ peak, $E^{\prime} / A^{\prime}$, and $E / E^{\prime}$ values showed statistically significant differences among RA patients. The results confirmed the presence of LV and RV impairment in RA.

In another Turkish study Seyfeli et al. [30] investigated RV diastolic function in RA and it's relationship with LV function and pulmonary involvement. 35 RA patients and 30 healthy controls were submitted to conventional Doppler (CE) and tissue Doppler echocardiography (TDE) to assess LV and RV systolic and diastolic function and to estimate maximal arterial systolic pulmonary pressure (PAP). To detect pulmonary involvement, pulmonary function tests and high-resolution computed tomography (HRCT) scans were performed in all RA individuals. An abnormal RV filling, as expressed by an inverted tricuspid (Tr.) E/ A ratio, was detected in $12(34 \%)$ of RA patients and in $2(7 \%)$ of controls $(\mathrm{p}<0.004)$. The prevalence of RV diastolic abnormalities was higher in RA patients examined by TDE, than by $\mathrm{CE}$ (RV annulus Em/Am ratio $<1$ in $31(89 \%)$ of patients) (p=0.002). 22 $(63 \%)$ RA subjects had abnormal HRCT findings. Pulmonary involvement with pulmonary hypertension (PHT) $(36 \pm 5 \mathrm{mmHg})$ was detected in $10(29 \%)$. In this group an increased RV annulus and basal Am wave, decreased Tr. E/A ratio and the decreased RV annulus $\mathrm{Em} / \mathrm{Am}$ ratio were statistically significant compared to RA patients with pulmonary involvement, who had normal PAP (19 $\pm 5 \mathrm{mmHg}$ : 12 [34\%] of 35), $\mathrm{p}=0.014, \mathrm{p}=0.006, \mathrm{p}=0.015$, $p=0.049$, respectively). The study revealed an impaired RV filling in a significant part of RA patients without overt heart failure. Impairment of RV diastolic function can be a predictor of subclinical myocardial and pulmonary involvement in RA.

The aim of the next Turkish study of Guyler et al. [31] was the assessment of $P$ wave dispersion (PWD) as a sign for the prediction of atrial fibrillation (AF) and it's relation to clinical and echocardiographic parameters in RA patients. 30 patients (mean age $49 \pm 10$ years) and 27 healthy controls (mean age $47 \pm 8$ years) were included in the study and electrocardiography and Doppler echocardiography was performed. Maximum and minimum $P$ wave duration were obtained from electrocardiographic measurements. PWD defined as the difference between maximum and minimum $P$ wave duration was calculated. Maximum $\mathrm{P}$ wave duration and PWD were higher in RA patients than in the controls $(p=0.031$ and $p=0.001$, respectively), however there were no significant correlation between PWD and disease duration $(r=0.375, p=0.009)$ and IVRT $(r=0.390, p=0.006)$. P wave duration 
and PWD was higher in RA patients than in the controls and was closely associated with the disease duration and LV diastolic dysfunction.

In the last Turkish study Canturk et al. [32] evaluated diastolic functions in patients with RA by assessment of propagation velocity and intraventricular dispersion of $\mathrm{E}$ wave velocity. 34 RA cases without evidence of cardiac disease and LV systolic impairment were enrolled in the study. Echocardiographic examinations were performed for the evaluation of diastolic dysfunction (DD) in all of them. Propagation velocity in RA patients was significantly lower than in the control group $(42 \pm 16 \mathrm{~cm} / \mathrm{s}, 54 \pm 15 \mathrm{~cm} / \mathrm{s}, \mathrm{p}=0.002)$. There was significant intraventricular dispersion of $\mathrm{E}$ wave velocity towards the cardiac apex in RA patients $(p<0.001)$ compared to the controls $(p=0.79)$. There was also a significant correlation between intraventricular dispersion of $\mathrm{E}$ wave velocity and diastolic dysfunction in the patients in which the duration of illness was longer than 10 years $(p<0.001)$. The authors concluded that an impaired LV relaxation in RA, correlated with the duration of the disease might be due to structural myocardial abnormalities. Furthermore, the combined use of propagation velocity and intraventricular $\mathrm{E}$ wave dispersion measurements can help in early determination of diastolic functions in RA patients.

The Russian publication of Krasnosel'sk $\breve{1}$ et al. [33] concerned the use of tissue dopplerography (TDG) in detection of myocardial involvement in RA by assessment of the velocity of myocardial movement. TDG was carried out in 22 patients with verified RA (age 33-66, mean age 45.2 \pm 7.4 years) and in 20 healthy volunteers (age 29-58, mean age 44.6 \pm 8.4 years). Modules of velocities of systolic and early diastolic peaks in LV wall at basal level and at levels of upper, middle and lower thirds were calculated in all patients. In some LV segments the results correlated with CRP and ESR in RA (r from 0.29 to 0.46 ) and were diffusely lowered in this disease, which on author's opinion reflected the participation of inflammatory process in development of asymptomatic diffuse myocardial damage in RA.

Gonzales-Juanatey et al. [34] examined Spanish population towards the frequency of echocardiographic and Doppler abnormalities in long-term treated RA patients without clinically overt heart disease. 47 such individuals, treated for at least 5 years with 1 or more DMARDs were recruited. Those with cardiovascular risk factors, cardiovascular or cerebrovascular events were excluded from the study. 47 healthy persons formed the control grouping patients with RA. The presence of aortic regurgitation (17\%) and tricuspid regurgitation $(17 \%)$ was higher than in the controls (15\% and 6\%, respectively). Pulmonary artery systolic pressure was higher in RA patients $(30.3 \pm 8.0 \mathrm{mmHg})$ compared to the in control subjects $(26.2 \pm 4.8)(\mathrm{p}=0.004)$. The incidence of pulmonary artery systolic pressure above $35 \mathrm{~mm} \mathrm{Hg}$ was significantly higher in RA patients $(21 \%$ versus $4 \%$ in controls; $p=0.03)$, diastolic dysfunction caused by impaired relaxation was more common in patients with RA $(66 \%)$ than in controls $(43 \%)(p=0.02)$ and more frequent in older patients. Extraarticular manifestations were more common in patients with RA in whom diastolic dysfunction was recognized $(p=0.05)$. This study confirmed a high frequency of LV diastolic dysfunction and pulmonary hypertension in patients with RA without evident cardiovascular disease.

In French patients Meune et al. [35] were determined the sensitivity and accuracy of tissueDoppler echocardiography (TDE) to assess myocardial contractility. The stimulus of this study was an observation that heart failure was one of the determinants of the excess mortality in RA patients. Consecutive 27 RA individuals with normal results of cardiac clinical examination were prospectively included and compared to 27 controls. All underwent conventional echocardiography, furthermore their systolic and diastolic strain 
rate (SR) were determined by TDE. When compared to the controls RA patients had increased LV mass $\left(99 \pm 24\right.$ vs $\left.80 \pm 25 \mathrm{~g} / \mathrm{m}^{2}, \mathrm{p}=0.009\right)$, and a trend towards left atrial enlargement ( $31 \pm 3$ vs $29 \pm 6 \mathrm{~mm}, \mathrm{p}=0.006)$. Fractional shortening and systolic SR did not differ between groups. Diastolic function, as estimated by the E/A Doppler velocity ratio was similar in both populations $(\mathrm{p}=0.18)$. However, diastolic $S R$ was strikingly reduced in patients with RA versus controls ( $3.7 \pm 1.3$ vs $5.5 \pm 1.1 \mathrm{~s}-1, \mathrm{p}<0.001)$. In 18 out of $27 \mathrm{RA}$ patients markedly reduced diastolic SR $(S R<4 s-1)$ was noted, too. None of the RA characteristics was associated with significant differences in TDE measurements. This study confirmed that TDE enable to identify impaired diastolic function in RA even in cases where it can not be detected by conventional measurements.

An Indian project of Uddayakumar et al. [36] was one of the in numerous studies on the prevalence of diastolic dysfunction in RA from Indian subcontinent. The aim of it was to evaluate LV filling abnormalities in RA patients without clinically evident cardiovascular manifestations and to search for their correlation with the disease duration. 45 such patients affected by RA diagnosed according to ARA criteria were selected and compared with age and sex matched control subjects. All were submitted to M-mode, two-dimensional and Doppler echocardiography. E, A, E/A ratio, IVRT, EF and fractional shortening were evaluated. In RA patients LV filling abnormalities were characterized by a reduced E/A ratio (mean [SD] 0.98 [0.22]) compared to the controls (1.09 [0.11]; p-value equals 0.004), prolonged IVRT (75.77 [8.12] ms versus 70.43 [2.94] ms; p-value equals 0.001) and increased late diastole flow velocity $(76.91[11.61] \mathrm{cm} / \mathrm{s}$ versus 70.11 [5.32] cm/s; p-value equals 0.001). A negative correlation was found between E/A ratio and the disease duration (Pearson correlation, $\mathrm{r}$ equals -0.56 , $\mathrm{p}$-value equals 0.001 ), indicating that diastolic dysfunction rate increased with disease duration. A strong correlation was also found between IVRT and the disease duration ( $\mathrm{r}$ equals $0.66, \mathrm{p}$-value equals 0.01 ) and between late diastolic flow velocity and the disease duration ( $\mathrm{r}$ equals 0.61 , p-value equals 0.001 ). The study confirms a high frequency of LV diastolic dysfunction characterized by impaired E/A ratio, prolonged IVRT and increased late diastole flow velocity in patients with RA without evident cardiovascular disease. The correlation between transmitral flow alteration and the disease duration suggests a progressive subclinical myocardial involvement with the disease progression. This may be relevant to high incidence of cardiovascular deaths observed in patients with RA.

Honda et al. [37] from Japan performed in 1981 studied cardiac function and vascular hemodynamics by non-invasive means in order to assess LV performance in 50 RA patients and 30 healthy controls. They did not find significant difference in LV end-diastolic dimension, LV posterior wall thickness, LV posterior wall excursion and mean posterior wall velocity between groups, yet the ejection fraction and ejection time/preejection period were significantly decreased. Hemodynamic status in patients with advanced RA in this study was characterized by mild tachycardia and a mild decrease of LV function, pericardial effusion was also noted in these individuals.

Echocardiography, a rapidly developing diagnostic procedure enable to examine heart structure and function in different clinical setting. It can be particularly useful in connective tissue diseases, such as rheumatoid arthritis. Generalized inflammatory process in these conditions affects heart muscle directly and through changes in tissue vasculature induced by cytokins and other inflammatory mediators of prothrombotic and atherogenic properties. Inflammatory vasculitis, another well-known complication of RA, especially of long duration, also contribute to heart damage in this disease. Cardiac muscle fibrosis and 
amyloidosis, that develop as further sequels of rheumatoid arthritis, add to cardiovascular system, especially diastolic function, impairment. Thrombus formation at heart valves with consecutive stenosis and/or regurgitations is an independent factor that disturb heart function in RA individuals.

Furthermore, one must not neglect the deleterious effects of drugs used to combat RA on cardiopulmonary system. Non-steroid anti-inflammatory agents with their water-retention and renal-toxic effects can induce or exacerbate hypertension and heart failure. So can cause corticosteroids, yet these are also known to strongly enhance atherogenesis and thrombosis. Methotrexate, the most popular and most effective of conventionally used DMARDs is able to induce lung fibrosis and therefore cause pulmonary hypertension, moreover it is renaltoxic. Chloroquine exerts proarrhytmic effect and cyklosporin induce hypertension. Gold salts, as well as D-penicillamine are also known to be capable of causing vasculitis and adversely affect patients' cardiovascular risk factor profile.

All these data clearly indicate to high risk of cardiovascular diseases in RA patients. Echocardiography in turn enables to detect early pathological changes of this kind and therefore can be helpful in prevention of the development of life-threatening complications. The results of studies presented in this topic confirm the utility of different echocardiography techniques in detecting heart damage in RA patients. Echocardiography visualizes for example decreased LV diastolic performance, as the earliest predictor of development of left ventricular heart failure, different kinds of valvular defects, pulmonary hypertension and the presence of pericardial effusion. The last one, although rarely clinically significant and only scarcely causing life-threatening complications is tamponade or constrictive pericarditis. Pericarditis is nevertheless present in about $30 \%$ of RA cases post-mortem [38]. It's appearance during patient's diagnostics could therefore be useful in recording cardiovascular involvement by RA, difficult or impossible to visualize by conventional methods. Another echocardiography application in RA is to investigate correlations between heart changes and the disease duration and activity, as well as with the patient's age. The discovery of such dependences can be helpful in prediction and therefore prevention of heart damage and perhaps other complications of RA.

Taken together - echocardiography is a chance for improving diagnostic methods in RA, yet further investigations are needed to work out techniques and medical standards in such patients. Hagendorff and Pfeiffer [39] focused on echocardiography application in modern diagnostics of connective tissue diseases, with special regard for rheumatoid arthritis. The authors concluded, that the prerequisites for successful diagnostic echocardiography in RA are the knowledge of modern echocardiographic techniques like tissue Doppler and contrast echocardiography and clinical experience with patients with rheumatoid arthritis. The standardization of procedures is important for reproducibility and comparability of results.

\section{References}

[1] Bacon PA, Gibson DG. Cardiac involvement in rheumatoid arthritis. An echocardiographic study. Ann Rheum Dis 1974; 33: 20-24.

[2] Prakash R, Atassi A, Poske R, Rosen KM. Prevalence of pericardial effusion and mitral valve involvement in patients with rheumatoid arthritis without cardiac symptoms. N Engl J Med 1973; 289: 597-600.

[3] Nomeir AM, Turner R, Watts E, Smith D, West G, Edmonds J. Cardiac involvement in rheumatoid arthritis. Ann Int Med 1973; 79: 800-806. 
[4] Mac Donald WJ Jr, Crawford MH, Klippel JH, Zvaifler N, O'Rourke R. Echocardiographic assessment of cardiac structure and function in patients with rheumatoid arthritis. Ann J Med 1977; 63: 890-896.

[5] Davia JE, Cheitlin MD, de Castro CM, Lawless O, Niemi L. Absence of echocardiographic abnormalities of the anterior mitral valve leaflet in rheumatoid arthritis. Ann Int Med 1975; 83: 500-502.

[6] Nomeir AM, Turner RA, Watts E. Cardiac involvement in rheumatoid arthritis. Followup study. Arthritis Rheum 1979; 25:561- 564.

[7] Mody GM, Stevens JE, Meyers OL. The heart in rheumatoid arthritis - a clinical and laboratory study. Q J Med 1987; 65: 921-928.

[8] Toumanidis ST, Papamichael CM, Antoniades LG, Pantelia MI, Saridakis NS, Mavrikakis ME, Sideris DA, Moulopoulos SD. Cardiac involvement in collagen diseases Eur Heart J 1995; 16: 257-262.

[9] Nagyhegyi G, Nadas I, Banyai F, Luzsa G, Geher P, Molnar J, Velics V, Gomor B, Weisz M, Antaloczy Z. Cardiac and cardiopulmonary disorders in patients with ankylosing spondylitis and rheumatoid arthritis. Clin Exp Rheumatol. 1988;6:17-26.

[10] Villecco AS, Ferri S, de Liberali E, Ferrari G, Marsigli R. Apport de l'echocardiographie au depistage des lesions cardiaques dans la polyarthrite rhumatoide. Revue $d u$ Rhumatisme 1983; 50: 187-193.

[11] Wislowska M, Sypuła S, Kowalik I. Echocardiographic findings, 24-hour electrocardiographic Holter monitoring in patients with rheumatoid arthritis according to Steinbrocker's criteria, functional index, value of Waaler-Rose titre and duration of disease. Clin Rheumatol 1998;17:369-377.

[12] Wislowska M, Sypuła S, Kowalik I. Echocardiographic findings and 24-h electrocardiographic Holter monitoring in patients with nodular and non-nodular rheumatoid arthritis. Rheumatol Int 1999; 18: 163-169.

[13] Barlow JB, Pocock WA. Mitral valve prolapsed, the specific billowing mitral leaflet syndrome, or an insignificant non-ejection systolic click. Am Heart J 1979; 97:277285.

[14] Leatham A, Brigden W. Mild mitral regurgitation and the mitral prolapsed fiasco. Am Heart J 1980; 99: 659-664.

[15] Wislowska M, Jaszczyk B, Kochmański M, Sypuła S, Sztechman M. Diastolic heart function in rheumatoid arthritis patients. Rheumatol Int 2008; 28: 513-519.

[16] Alpaslan M, Onrat E, Evcik D. Doppler echocardiographic evaluation of ventricular function in patients with rheumatoid arthritis. Clin Rheumatol 2003; 22: 84-88.

[17] Di Franco M, Paradiso M, Mammarella A, Paoletti V, Labbadia G, Coppotelli L, Taccari E, Musca A. Diastolic function abnormalities in rheumatoid arthritis. Evaluation by echo Doppler transmitral flow and pulmonary venous flow: relation with duration of disease. Ann Rheum Dis. 2000;59:227-229

[18] Levendoglu F, Temizhan A, Ugurlu H, Ozdemir A, Yazici M. Ventricular function abnormalities in active rheumatoid arthritis: a Doppler echocardiographic study. Rheumatol Int. 2004;24:141-146.

[19] Maione S, Valentini G, Giunta A, Tirri R, Giacummo A, Lippolis C, Arnese M, de Paulis A, Marone G, Tirri G. Cardiac involvement in rheumatoid arthritis: an echocardiographic study. Cardiology 1993; 83: 234-239. 
[20] Corrao S, Sallì L, Arnone S, Scaglione R, Pinto A, Licata G. Echo-Doppler left ventricular filling abnormalities in patients with rheumatoid arthritis without clinically evident cardiovascular disease. Eur J Clin Invest. 1996;26:293-297.

[21] Montecucco C, Gobbi G, Perlini S, Rossi S, Grandi AM, Caporali R, Finardi G. Impaired diastolic function in active rheumatoid arthritis. Relationship with disease duration. Clin Exp Rheumatol. 1999;17:407-412.

[22] Voipio-Pulkki LM, Sarate M. Left ventricular function in rheumatoid arthritis. Eur Heart J 1987; 8 (Supplement J), 105-107.

[23] Mustonen J, Laakso M, Hirvonen T, Mutru O, Pirnes M, Vainio P, Kuikka JT, Rautio P, Länsimies E. Abnormalities in left ventricular diastolic function in male patients with rheumatoid arthritis without clinically evident cardiovascular disease. Eur J Clin Invest. 1993;23:246-53.

[24] Kozakova M, Hradec J, Petrasek J, Kolbel F, Urbanova M, Dostal C. Cardiac involvement in progressive polyarthritis - an echocardiographic study. Cor Vasa 1985; 27: 54-59.

[25] Alusik S, Skalicka H. Cardiac involvement in rheumatoid arthritis - an echocardiographic study. "R", 1991, 21: 17-21.

[26] Arslan S, Bozkurt E, Sari RA, Erol MK. Diastolic function abnormalities in active rheumatoid arthritis evaluation by conventional Doppler and tissue Doppler: relation with duration of disease. Clin Rheumatol. 2006 ;25:294-299.

[27] Rexhepaj N, Bajraktari G, Berisha I, Beqiri A, Shatri F, Hima F, Elezi S, Ndrepepa G. Left and right ventricular diastolic functions in patients with rheumatoid arthritis without clinically evident cardiovascular disease. Int J Clin Pract. 2006;60:683-688.

[28] Yazici D, Tokay S, Aydin S, Toprak A, Inanc N, Khan SR, Fak AS, Direskeneli H. Echocardiographic evaluation of cardiac diastolic function in patients with rheumatoid arthritis: 5 years of follow-up. Clin Rheumatol. 2008;27:647-650.

[29] Birdane A, Korkmaz C, Ata N, Cavusoglu Y, Kasifoglu T, Dogan SM, Gorenek B, Goktekin O, Unalir A, Timuralp B. Tissue Doppler imaging in the evaluation of the left and right ventricular diastolic functions in rheumatoid arthritis. Echocardiography. 2007;24:485-493.

[30] Seyfeli E, Guler H, Akoglu S, Karazincir S, Akgul F, Saglam H, Seydaliyeva T, Yalcin F. Right ventricular diastolic abnormalities in rheumatoid arthritis and its relationship with left ventricular and pulmonary involvement. A tissue Doppler echocardiographic study. Int J Cardiovasc Imaging 2006; 22: 745-754.

[31] Guyler H, Seyfeli E, Sahin G, Duru M, Akgul F, Saglam H, Yalcin F. P wave dispersion in patients with rheumatoid arthritis: its relation with clinical and echocardiographic parameters. Rheumatol Int 2007;27:813-818.

[32] Canturk F, Yazici M, Alayli G, Menekse EB, Demircan S, Ibrahimli F, Menekse S. Combined use of propagation velocity and intraventricular dispersion of $\mathrm{E}$ wave velocity for the evaluation of diastolic functions in patients with rheumatoid arthritis. Int J Cardiovasc Imaging. 2006;22:369-376.

[33] Krasnosel'skĭ MI, Bratanova MZ, Polupan AA, Tsurko VV. Tissue dopplerography of the myocardium in diagnosis of myocardial involvement in patients with rheumatoid arthritis. Kardiologiia. 2007;47:59-62.

[34] Gonzalez-Juanatey C, Testa A, Garcia-Castelo A, Garcia-Porrua C, Llorca J, Ollier WE, Gonzalez-Gay MA. Echocardiographic and Doppler findings in long-term treated 
rheumatoid arthritis patients without clinically evident cardiovascular disease. Semin Arthritis Rheum. 2004;33:231- 238.

[35] Meune C, Wahbi K, Assous N, Weber S, Kahan A, Allanore Y. Myocardial dysfunction in rheumatoid arthritis: a controlled tissue-Doppler echocardiography study. J Rheumatol. 2007;34:2005-2009.

[36] Udayakumar N, Venkatesan S., Rajendiran C. Diastolic function abnormalities in rheumatoid arthritis: relation with duration of disease. Singapore Med J. 2007;48:537542.

[37] Honda M, Muraki N, Takahari O, Nohira K, Matsui N, Hirai A. Cardiac function and vascular hemodynamics in rheumatoid arthritis. Revue du Rhumatisme 1981; (numero special Juin) 0320.

[38] Leibowitz WB. The heart in rheumatoid arthritis (rheumatoid disease). A clinical and pathological study of 62 cases. Ann Int Med 1963; 58: 102-123.

[39] Hagendorff A, Pfeiffer D. Echocardiographic functional analysis of patients with rheumatoid arthritis and collagen diseases. Z Rheumatol. 2005; 64: 239-248. 


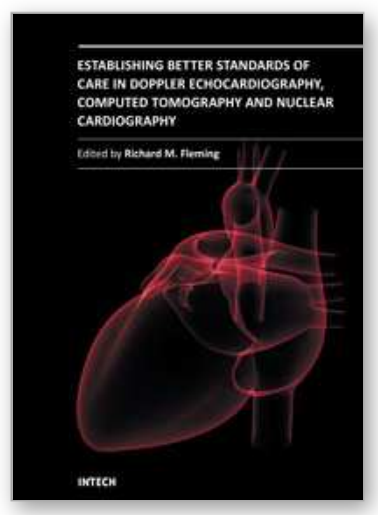

\author{
Establishing Better Standards of Care in Doppler \\ Echocardiography, Computed Tomography and Nuclear \\ Cardiology \\ Edited by Dr. Richard M. Fleming
}

ISBN 978-953-307-366-8

Hard cover, 260 pages

Publisher InTech

Published online 13, July, 2011

Published in print edition July, 2011

Since the introduction of Doppler Echocardiography, Nuclear Cardiology and Coronary CT imaging, clinicians and researchers have been searching for ways to improve their use of these important tools in both the diagnosis and treatment of heart disease. To keep up with cutting edge improvements in these fields, experts from around the world have come together in this book to provide the reader with the most up to date information to explain how, why and when these different non-invasive imaging tools should be used. This book will not only serve its reader well today but well into the future.

\title{
How to reference
}

In order to correctly reference this scholarly work, feel free to copy and paste the following:

Małgorzata Wisłowska (2011). Echocardiography in Rheumatoid Arthritis (RA), Establishing Better Standards of Care in Doppler Echocardiography, Computed Tomography and Nuclear Cardiology, Dr. Richard M. Fleming (Ed.), ISBN: 978-953-307-366-8, InTech, Available from:

http://www.intechopen.com/books/establishing-better-standards-of-care-in-doppler-echocardiographycomputed-tomography-and-nuclear-cardiology/echocardiography-in-rheumatoid-arthritis-ra-

\section{INTECH}

open science | open minds

\section{InTech Europe}

University Campus STeP Ri

Slavka Krautzeka 83/A

51000 Rijeka, Croatia

Phone: +385 (51) 770447

Fax: +385 (51) 686166

www.intechopen.com

\section{InTech China}

Unit 405, Office Block, Hotel Equatorial Shanghai

No.65, Yan An Road (West), Shanghai, 200040, China

中国上海市延安西路65号上海国际贵都大饭店办公楼 405 单元

Phone: +86-21-62489820

Fax: $+86-21-62489821$ 
(C) 2011 The Author(s). Licensee IntechOpen. This chapter is distributed under the terms of the Creative Commons Attribution-NonCommercialShareAlike-3.0 License, which permits use, distribution and reproduction for non-commercial purposes, provided the original is properly cited and derivative works building on this content are distributed under the same license. 